\title{
Sublingual immunotherapy in allergic rhinitis: indications, efficacy and safety
}

\author{
Harsha H. Kariyawasam', Giuseppina Rotiroti', Douglas S. Robinson² \\ Royal National Throat Nose Ear Hospital London, University College London, London, United Kingdom \\ Leukocyte Biology Section, MRC and Asthma UK Centre for Allergic Mechanisms in Asthma, Imperial College London, London, \\ United Kingdom
}

Rhinology 51: 000-000, 2013 DOI: 10.4193/Rhino.11.268

*Received for publication: December 29, 2011 Accepted: July 6, 2012

\section{SUMMARY}

Sublingual immunotherapy (SLIT) is now an established treatment for allergic rhinitis. Whilst several systematic reviews have now confirmed clinical efficacy and safety, recent analysis also confirms that therapeutic benefit persists for several years after the completion of SLIT. Such findings, along with the obvious ease of administration of sublingual preparations, have clearly promoted SLIT as an attractive option for treatment of allergic rhinitis resistant to medical treatment. This article reviews the development, efficacy and safety of SLIT in allergic rhinitis and the current indications for its use.

Key words: allergic rhinitis, sublingual, immunotherapy

\section{Allergic rhinitis}

Allergic rhinitis affects up to $25 \%$ of Western populations. It is a major health burden, not only as a result of the high prevalence and the associated economic costs, but also because of the significant effect the disease has on individual wellbeing, performance at school or work and overall quality of life ${ }^{(1)}$. The Allergic Rhinitis and its Impact on Asthma (ARIA) document has moved on from the terms seasonal or perennial allergic rhinitis, which does not take into account warmer climates where pollens can be present year-round. Instead it defines the disease according to symptom duration and severity and thus allows a more global classification of the disease and immediately links allergic rhinitis to the adverse effect it has on function and wellbeing in an individual. This concept is outlined in Figure 1, as adapted from the ARIA guidelines ${ }^{(1)}$. Such a practical classification allows the severity of disease to be matched to an appropriate stage of pharmacotherapy.

\section{Clinical symptoms}

The occurrence of two or more nasal symptoms of congestion, rhinorrhoea and nasal irritability (sneeze/itch) in relation to allergen exposure along with demonstration of IgE-specific sensitisation is diagnostic of allergic rhinitis. Ocular symptoms are common, particularly so in pollen-related rhinitis and the term allergic rhinoconjunctivitis may be more appropriate. In Europe, predominant sensitisation is to the house dust mite (HDM) species Dermatophagoides pteronyssinus, with a median prevalence of $21.7 \%$ followed by grass pollen (predominantly Timothy grass or Phleum pratense) at $16.9 \%{ }^{(2)}$. Individuals with persistent symptoms that are moderate-severe should be considered for treatment with immunotherapy. As such, particularly in the UK, failure of maximal pharmacotherapy (topical corticosteroids and antihistamines) should suggest immunotherapy as a treatment option. The treatment approach is summarised in Figure 2.

\section{Immunotherapy}

Allergen specific immunotherapy (SIT) involves repeated administration of the allergen causing disease. The therapeutic aim is to induce sustained immunological tolerance to the specific allergen such that subsequent exposure fails to induce clinical symptoms. This is termed desensitisation and with time can alter the clinical course of the disease ${ }^{(1)}$. Double-blind randomised placebo-controlled (DBRPC) clinical studies and robust meta-analysis have confirmed efficacy in allergic rhinitis. As a result, the World Health Organisation ${ }^{(3)}$, the European Academy of Allergology and Clinical Immunology ${ }^{(4)}$, the World Allergy 
Organisation position paper ${ }^{(5)}$, the British Society for Allergy and Clinical Immunology ${ }^{(6)}$ and the American Academy of Allergy, Asthma and Immunology and the American College of Allergy Asthma and Immunology ${ }^{(7)}$ consensus documents all recommend SIT for individuals with moderate to severe allergic rhinitis.

SIT has traditionally been administered using subcutaneous injections (termed subcutaneous immunotherapy or SCIT) and at present is the only form of SIT approved by the Food and Drug Administration (FDA) agency in the USA. However, the necessity for frequent hospital visits, sometimes for 3 years or more is inconvenient, the discomfort of injections such as local pain, itch and swelling are relatively common and the more serious adverse events of anaphylaxis and death still occur. Such difficulties have led to a focus on the development of modified extracts or alternative routes of delivery. Sublingual immunotherapy (SLIT) offers an alternative approach, which can overcome many of the concerns associated with SCIT. Several DBRPC studies now confirm therapeutic efficacy. As a result, SLIT is an attractive therapeutic option.

\section{Immunotherapy in allergic rhinitis}

Despite maximal medical therapy using nasal steroids, anti-histamines, leukotriene inhibitors and nasal douching, a proportion of patients with allergic rhinitis are left with severe and debilitating symptoms ${ }^{(8)}$. In this severe failure to respond group, subcutaneous immunotherapy (SCIT) has provided effective clinical intervention with sustained clinical improvement, even after discontinuation of treatment. Although modified extracts with reduced IgE binding such as chemically modified extracts (allergoids) improve safety and reduced the number of injections, and modified recombinant allergens or peptide fragments are in development, some patients find injected therapy inconvenient. SLIT offers improved safety and convenience in that it can be used away from a hospital setting.

\section{Major aeroallergens in allergic rhinitis}

\section{Grass Pollen}

Grass pollen is estimated to affect $90 \%$ of hay fever sufferers and grass-specific immunotherapy accounts for one third of specific immunotherapy undertaken in Europe ${ }^{(3)}$. It is directed against the common grasses in the Pooideae subfamily. The very similar morphology between the pollens in the subfamily leads to $\lg \mathrm{E}$ cross- reactivity which makes it difficult to determine which pollen is responsible for sensitisation ${ }^{(9)}$, although geographical location and a pollination calendar maybe helpful. SLIT products specific to one grass and mixed grasses are currently in clinical use and discussed later.

\section{Tree Pollen}

Tree pollens implicated in allergic rhinitis are extensive and include alder (Alnus), birch (Betula), willow (Salix), poplar (Populus), cedar (Cedrus), hazel (Corylus), hornbeam (Carpinus), horse chestnut (Aesculus), pine (Pinus), plane (Platanus), linden/lime (Tilia) and olive (Olea). In northern Europe birch pollen is the most prevalent form of tree induced allergic rhinitis, accounting for up to $15-20 \%$ of individuals.

\section{Weeds}

Ragweed (Ambrosia) and mugwort (Artemisia) are both commonly implicated in weed-induced allergic rhinitis, and have almost identical pollination season towards late July onwards. Parietaria species of the Urticaceae (nettle) family can thrive particularly in Mediterranean regions, sometimes all year round such as in Southern Italy leading to perennial symptoms of allergic rhinitis.

\section{House Dust Mite (HDM)}

Dermatophagoides pteronyssinus (D.pt) (European mite) and Dermatophagoides farinae (D.f) (American mite) are the most common mite species present world-wide. Although other mites maybe relevant in some Mediterranean populations, in Northern Europe sensitisation to the major allergen in D.pt, Der p1, the main immuno-dominant protein, leads to up to $80 \%$ of allergic reactions in HDM sensitised individuals. Whilst D.f is less common in Europe, its major allergen Derf1, shows 82\% homology to Der $p 1$ and is also a potent inducer of IgE mediated allergic inflammation. The main allergen proteins are found in high concentration in the dust mite faeces and outlast the life of the mite itself. SLIT formulations with HDM extract incorporate standardized D.pt and D.f allergen.

\section{Sublingual immunotherapy development}

It was Leonard Noon that demonstrated the efficacy of subcutaneous injections of pollen allergen for hayfever in 1911 and the progress made in the last hundred years has been reviewed in detail recently, as part of the centenary celebrations of immunotherapy ${ }^{(10)}$. The clinical benefits of immunotherapy were obvious from very early on and led to the establishment and maintenance of subcutaneous immunotherapy (SCIT) as the dominant form of immunotherapy for allergic rhinitis. However, definite data demonstrating the clinical efficacy of SCIT in the form of a DBRPC study only appeared in 1965, specific to ragweed induced allergic rhinitis ${ }^{(11)}$. This was a landmark study that brought to an end an era of unsupported clinical claims. The almost epidemic increase in the proportions of allergic patients that could benefit from immunotherapy was apparent and since then several well-deigned studies have demonstrated the clear benefits of treatment with SCIT and meta-analysis confirms significant clinical efficacy ${ }^{(12)}$.

However, the risks associated with SCIT had become very apparent to practising clinicians and a report by the Committee 


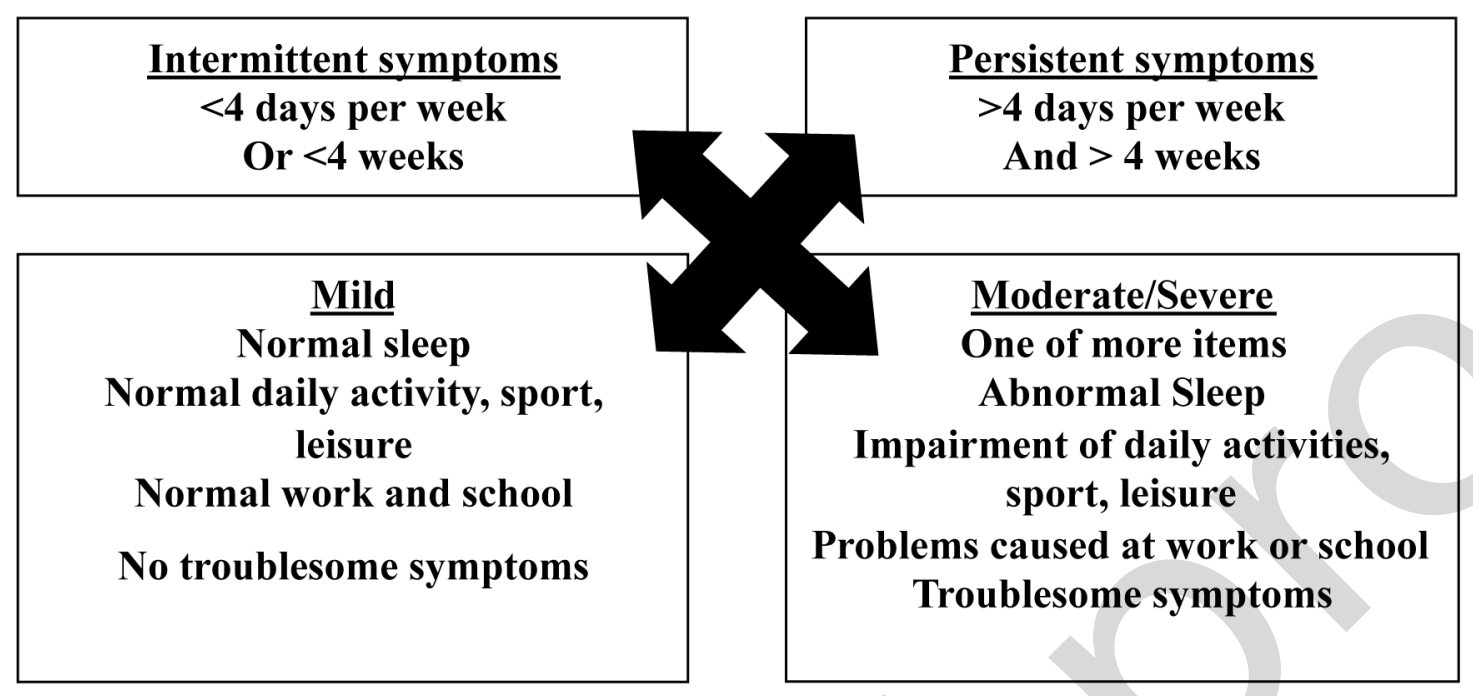

Figure 1. Classification of allergic rhinitis (adapted and modified from the ARIA guidelines). Allergic rhinitis is classified on the basis of duration and severity of symptoms in the ARIA document. This is a practical and clinically relevant classification which dictates the stage of pharmacotherapy that should be instigated. Patients with moderate-severe disease that is persistent should be considered for immunotherapy, particularly if they have failed maximal pharmacotherapy.

on Safety of Medicines (UK) in 1986 further highlighted safety concerns by reporting on SCIT-associated deaths. As a result, strict restrictions were placed on how and where SCIT could be undertaken in the UK, and the report pressed for the safety of immunotherapy to be revaluated world-wide. The required time commitment for hospital attendance at specialist allergist centres undertaking SCIT was inconvenient for patients and the need for significant staffing resources and hence cost to support such services, added further burden to SCIT. Thus, the urgent development of safer, more cost-effective and less intensive forms of immunotherapy was urgently needed. As a result, interest in oral delivery of allergen was reignited.

The first DBRPC trial with sublingual drops in HDM induced allergic rhinitis was undertaken in $1986^{(13)}$. It was a cross-over study and used a droplet formulation of HDM extract. The lowest concentration of serially diluted allergen extract that failed to further increase wheal size on intradermal administration, thus a HDM concentration specific to each participant, was used as the SLIT dose. A volume of $0.1 \mathrm{ml}$ was taken sublingually four-times a day. Despite only limited patient numbers that completed the active treatment arm, significant improvements in nasal air flow and less reactivity to nasal HDM provocation was demonstrated. Such encouraging findings pressed the need for further detailed studies.

The more recent trials have been robust in design and outcome evaluation. As such it is only now that acceptance of SLIT as a serious alternative to SCIT is emerging, and has rapidly become the most prescribed form of immunotherapy in Europe. Recent positive DBRPC studies in the United States have also begun to alter the American stance on SLIT ${ }^{(14,15)}$ and it is hoped that FDA approval will be forthcoming in the near future ${ }^{(16)}$.

\section{Indications for immunotherapy}

A clear history relating exposure to a specific allergen inducing clinical symptoms consistent with that of allergic rhinoconjunctivitis.

Confirmation of sensitisation to allergen elicited in clinical history through either skin prick test or specific lgE in serum.

Complete future allergen avoidance is not possible.

Failure to respond to maximal medical therapy (although SLIT 


\section{Confirm Diagnosis of Allergic Rhinitis (history and directed investigations)}

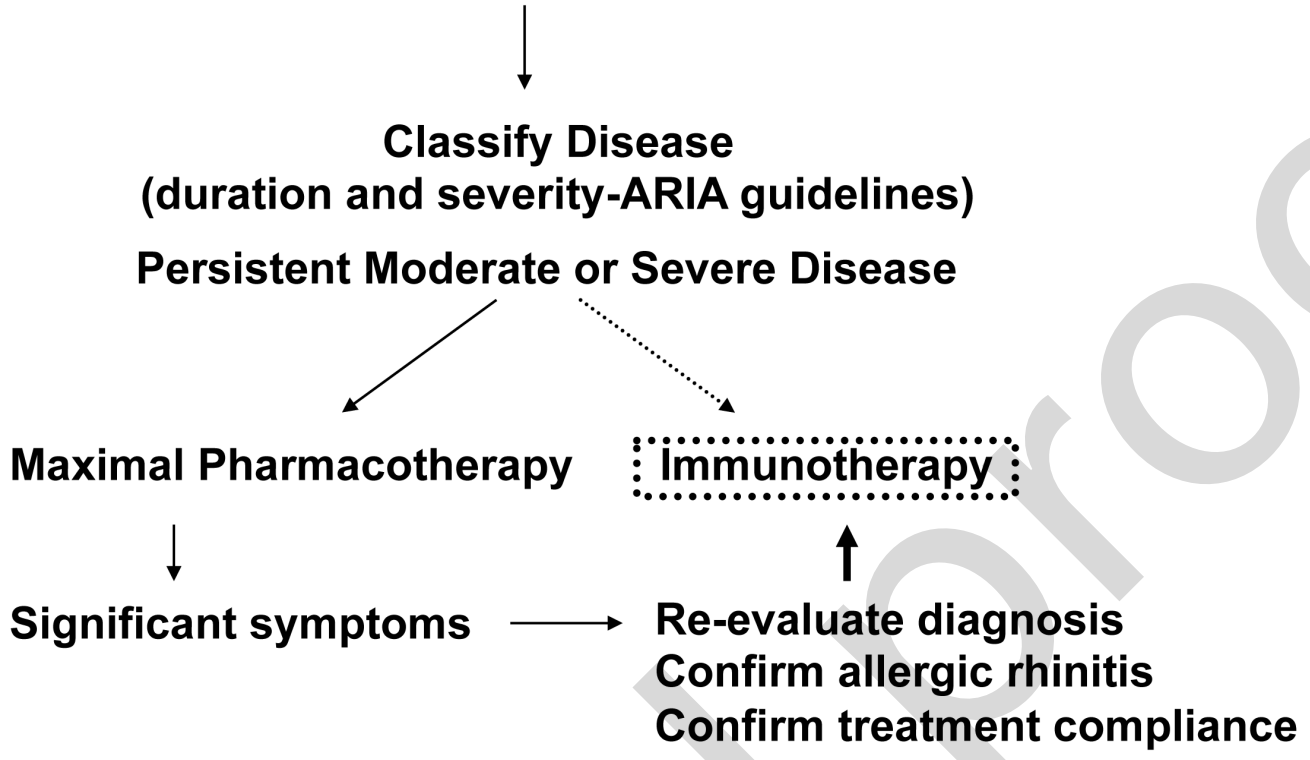

Figure 2. Immunotherapy in allergic rhinitis. Immunotherapy is currently considered for individuals with moderate-severe persistent allergic rhinitis, usually when failing maximal pharmacotherapy. Given the convenience of SLIT and excellent safety profile, immunotherapy may even be indicated early on, along-side pharmacotherapy (as indicated by the dotted line). As allergen-specific tolerance develops, a gradual step-down in medication can often be achieved.

can be used as initial therapy at the discretion of the clinician) ${ }^{(5)}$.

\section{Contraindications for sublingual immunotherapy}

Oral mucosal disease (oral lichen planus, ulceration or oral mycosis for example).

Malignancy, autoimmune disease, immune complex disease, severe immune deficiency or concurrent immunosuppressive administration.

Pregnancy (although patients can continue therapy in the absence of any systemic side effects at the discretion of the clinician).

Co-medication with a beta-blocker (including eye drops).

Anaphylaxis to the SCIT formulation of allergen (cautious use).

\section{SLIT formulations}

Sublingual aqueous droplets

Allergen drops are placed underneath the tongue and held for several minutes and subsequently swallowed with the saliva.

This allows rapid allergen absorption into the extensive vascular and lymphoid system of the buccal mucosa. There is probably a role for gastrointestinal absorption of allergen as analysis of clinical efficacy data suggests less benefit with sublingual 'hold and spit' regimes ${ }^{(17)}$. As such 'sublingual hold-swallow' is recommended and currently used.

\section{Sublingual tablets}

The preparations are formulated to be rapidly absorbed into the sublingual mucosa and subsequent swallow a minute later of saliva may allow any unabsorbed allergen to be sampled by the gut associated lymphoid tissue (GALT). The extensive distribution of GALT and the potency by which this lymphoid organ can induce antigenic tolerance is important. As such oral enteric coated tablet preparations of allergen that are directly swallowed and can bypass the stomach with $\mathrm{pH}$ dependent release of allergen in the small intestine have also been investigated in the past, but were associated with significant gastrointestinal symptoms ${ }^{(18,19)}$. 


\section{SLIT clinical efficacy}

The early studies in relation to SLIT lacked robustness to draw any definite conclusions but suggested clinical efficacy and adequate safety to pursue larger and more rigorous studies. Rapid expansion in such studies has provided definite data for meta-analysis/systematic review (i.e. considered Grade A based evidence). In the initial assessment combining both children and adults by Wilson and colleagues in 2003, 22 DBRPC studies up to 2002 fulfilled the Cochrane Foundation methodology confirmed criteria for inclusion. The clinical response in 979 individuals was evaluated ${ }^{(17)}$. Only 5 studies were in children. There was significant trial heterogeneity that made analysis less straightforward. There were 6 studies in relation to house-dust mite, with 4 studies for grass and 5 studies with Parietaria. Only 2 studies focused on olive tree and there was 1 study each for cat, tree and the genus Cupressus (trees and shrubs native to warm temperate regions in the northern hemisphere). A total of 17 studies used sublingual drops with 'hold and swallow'; 3 with 'hold and spit' and only 2 studies evaluated sublingual tablets. There was further significant study heterogeneity in terms of disease evaluation. Nevertheless, a significant reduction in symptoms $(p=0.002)$ and medication usage $(p=0.0003)$ was seen, with no information as to whether any particular allergen extract or formulation was particularly more effective. However, there was no significant demonstration of benefit for children at this time, probably a reflection of the limited total numbers evaluated. Importantly SLIT was confirmed as safe, with only minor adverse events reported.

Efficacy of SLIT in children 3-18 years of age was confirmed by meta-analysis in $2006^{(20)}$. The authors evaluated only 10 out of 70 articles identified from 1990 to 2004 and confirmed a reduction in symptoms $(p=0.02)$ and medication use $(p=0.03)$, with the suggestion that treatment duration more than 18 months was relevant. Despite heterogeneity of the trials evaluated, pollen SLIT appeared more effective than mite desensitisation therapy.

There has been such a rapid revival in the interest in SLIT that further meta-analysis in 2010 incorporates a total of 49 studies up until August $2009^{(21)}$. In addition to evaluating the overall clinical efficacy, the authors here also investigated key immunological responses implicated with tolerance induction and sustenance. The significant findings here were that studies with grass pollen were now increased to 23 , house dust mite to 8 , whilst Parietaria was 5, tree was 9 ( 2 birch, 2 olive, 3 cypress and 2 mixed trees), and there was only 1 study in relation cat allergen desentisation. There were 15 studies specific to children. The reduction in clinical symptoms $(p<0.00001)$ and medication use $(p<0.00001)$ was highly significant. The greater number of total patients allowed more robust subgroup analysis. Treat- ment effect in children was not shown as equivalent to adults. Subgroup analysis confirmed symptom score reduction at $p<$ 0.00001 for grass pollen SLIT from a total of 23 studies, whilst HDM and tree pollen SLIT were still significant at $p=0.02$ only. Both the droplet and tablet forms of SLIT were highly effective $(p<0.00001)$. In addition further meta-analysis has confirmed significant clinical benefit in the reduction of allergic conjunctivitis symptoms with SLIT ${ }^{(22)}$.

Meta-analysis dedicated to SLIT with HDM identified a total of 8 studies that were suitable for review ${ }^{(23)}$. However, it failed to identify significant clinical efficacy in terms of symptom reduction and medication use when children and adult studies were evaluated separately. Pooled analysis only led to a statistically significant outcome for symptom improvement $(p=0.02)$ and medication reduction $(p=0.04)$. Heterogeneity of study protocols and different SLIT preparations, along with relatively small numbers of patient at a total of 382 will have also affected analysis outcomes. As such, the up-take of SLIT for HDM induced allergic rhinitis has been limited, although several DBRPC studies using more refined SLIT products with standardised protocols are in progress, and the results are eagerly awaited.

\section{Immunological tolerance induction by SLIT}

Allergen specific $\lg E$ is present on the surface of mucosal mast cells (MC) bound by the high affinity IgE receptor FceRI. Allergen impacts against the nasal mucosa and is recognised and bound by the allergen-specific lgE. The allergen cross-linking of two or more FcERI-IgE complexes leads to MC activation, degranulation and subsequent release of inflammatory mediators that initiate the immediate rhinitis symptoms of sneezing, rhinorrhoea and congestion (i.e. induction of acute inflammation). Professional antigen presenting cells (called dendritic cells or DCs) also take up allergen and present processed allergen peptide to naïve $T$ cells, which then undergo activation and differentiation to Th2 cells that drive allergic inflammation. This leads to the further generation and release of important cytokines such as interleukin (IL)-4 (promotes further lgE synthesis from B cells), IL-5 (recruits eosinophils to the nasal tissue and promotes eosinophil survival) and IL-13 (a powerful cytokine implicated in driving several aspects of airway allergy). Thus chronic inflammation ensues that is frequently exacerbated with recurrent allergen exposure.

We are still in the process of understanding the exact mechanisms by which SLIT confers therapeutic efficacy and has recently been reviewed in detail ${ }^{(24)}$. The current concepts are outlined in basic form in Figure 3. The oral mucosa is a site of natural immunological tolerance. The local dendritic cells (termed oral Langerhans cells or oLCs) can directly up-take allergen. This step maybe dose-dependent, with a higher allergen dose leading to a more enhanced response, explaining why high-dose allergen SLIT seems to confer greater clinical efficacy ${ }^{(25)}$. This leads to an 
Low dose allergen delivery via natural exposure

Chronic Inflammation

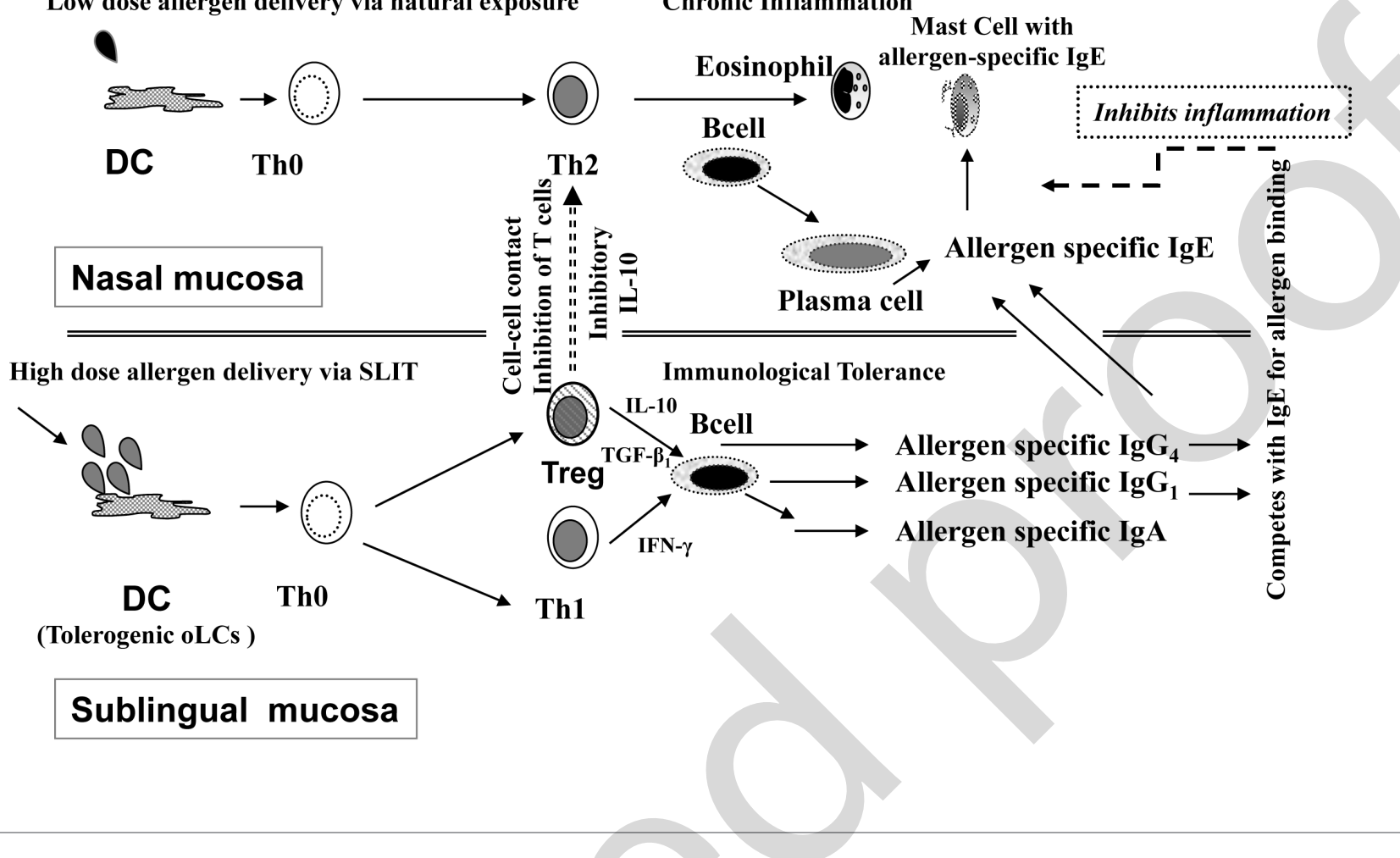

Figure 3. Mechanisms of sublingual immunotherapy (SLIT). In allergic rhinitis, low doses of aeroallergens drive the lgE mediated allergic reaction in the nasal mucosa. The allergic pathway is predominantly driven by the Th2 subset of T cells, which are promoted by dendritic cells (DC) of the nasal mucosa. In contrast, when high dose allergen is delivered via the sublingual mucosa, the local DCs termed oral Langerhans cells (oLCs), which are intrinsically tolerogenic, drive naïve (Th0) T cells into the Th1 subset along with the generation of T cells (collectively termed T regulatory cells or Tregs) that suppress Th2/Th1 cells through both cell-cell contact mediated inhibition and generation of the anti-inflammatory cytokines IL-10 and transforming growth factor (TGF)- $\beta 1$. In addition, the generation of allergen specific lgG1/lgG4 functional inhibitory antibodies that can compete with and block key lgE-allergen binding mediated signalling events is important.

altered oLC- naïve T cell interaction, directly in the sublingual mucosal surface and also on reaching the draining lymphoid tissue of the mouth. As such, the generation of an altered (less allergenic) T cell phenotype is favoured (i.e. Th1 cells) and T cells that regulate inflammation (termed $T$ regulatory cells or Tregs) are generated. Production of key anti-inflammatory cytokines (such as IL-10 and TGF- $\beta_{1}$ ) will attenuate several aspects of airway inflammation and alter $B$ cell responses. The latter is manifested by sustained increases in allergen-specific $\lg G_{1}$ and $\lg _{4}$ antibodies that can functionally 'block' key allergic events such as IgE binding of allergen (Figure 3). Such antibodies have been demonstrated as surrogate markers of immunological tolerance after grass-pollen immunotherapy ${ }^{(26)}$. Within a short period such potent immunological events begin to counteract and attenuate key steps of the IgE mediated allergic cascade. Allergen tolerance is achieved and the clinical translation is a reduction of symptoms and medication use despite continued inhalational allergen exposure.

\section{SLIT products}

We will discuss selected registered products that have current market dominance in the UK. The list is not exhaustive given the space constraints of the article.

\section{Sublingual Tablets}

SLIT in tablet formulation allows the delivery of a more uniform high allergen dose and enhanced local application compared to sublingual droplet formulations that rapidly disperse. This will probably enhance local uptake by mucosal antigen presenting cells (dendritic cells) of the sublingual mucosa and potentially offer greater clinical efficacy. As such, much of the more recent focus has been into the development of tablet formulations of SLIT, particularly in relation to treatment of grass pollen induced seasonal allergic rhinitis. 


\section{Grazax}

Grazax (ALK Abelló, Hørsholm, Denmark) is a sublingual immunotherapy tablet that is formulated as a compact lyophilisate form containing standardised allergen extract of pollen from Timothy grass (Phleum pratense). The allergen dose is 75,000 standardised quality (SQ-T) units per tablet and is taken on a daily basis. The lyophilisate is placed underneath the tongue for at least one minute and the saliva then swallowed. Optimal benefit is seen when taken at least 8 weeks before the onset of the pollen season ${ }^{(27)}$. Robust efficacy and safety data from 15 randomised DBRPC and 10 observational studies (involving over 2000 adults and 500 children) is available ${ }^{(28)}$. The product has been launched in Europe. Grazax is licensed for adults and children 5 - 16 years of age with grass-pollen induced allergic rhinitis. Timothy grass (Phleum pratense) demonstrates strong cross-reactivity with other Pooideae members such as rye (Lolium species), meadow fescue (Festuca species), orchard/ cocksfoot (Dactylis species), redtop/bent/velvet (Agrostis species), sweet vernal (Anthoxanthum species) and bluegrass/June (Poa species). It will have some cross-reactivity with Johnson grass (Sorghum halepense). These are all major pollen aeroallergens. Phase III studies with sublingual Phleum pratense 75,000 SQ-T have recently been completed in North America ${ }^{(15)}$, a necessary measure if FDA approval is to be sought.

The first trial was a dose finding study with initiation of treatment 8 weeks prior to the grass pollen season, and it was shown that clinical benefits were most apparent in the study arm that received the highest dose of allergen at 75,000 SQ-T units (15mcg) ${ }^{(25)}$. The subsequent GT-08 study used 75,000 SQ-T units a day, starting therapy 16 weeks prior to the grass-pollen season, and continued uninterrupted for 3 years with a further 2 years double-blinded follow up. All subjects had moderate to severe seasonal allergic rhinitis from grass pollen. Both the interim analysis at 2 years ${ }^{(29)}$ and at study completion after 3 years of treatment ${ }^{(30)}$ confirmed significant improvement in the rhinoconjunctivitis symptom and medication scores along with improvement in rhinoconjunctivitis quality of life. Commencement of treatment at least 8 weeks or longer before the pollen season is needed to confer greater treatment efficacy ${ }^{(27)}$. Importantly, clinical tolerance to grass pollen was maintained 2 years after cessation of treatment with the combined symptom and medication scores demonstrating a $33 \%$ reduction in the group that had received active treatment compared to placebo (31). A biomarker of immune tolerance $\operatorname{lgG}_{4}$ specific to grass allergen progressively increased in serum during the active study period, and this increase was sustained after cessation of therapy albeit at slightly lower levels, coinciding with the observation of clinical tolerance to grass pollen for the 2 years post treatment (31). Reassuringly no safety issues have been raised in this follow up period.

\section{Oralair}

Oralair" (Stallergenes, France) is a five-grass sublingual tablet for grass-pollen induced rhinoconjunctivitis. It is indicated for adults and children from 5 years onwards. The active allergen is a composite of 5 grass species: perennial rye grass (Lolium perenne), meadow grass (Poa pratensis), timothy grass (Phleum pratense), cocksfoot (Dactylis glomerata) and sweet vernal grass (Anthoxanthum odoratum). A dose ranging study in adults administered the 5-grass sublingual tablet (placebo versus $100-I R, 300-I R$ or 500IR) on a daily basis, commencing 4 months before the onset of the grass pollen season and till end of the season. It was confirmed that both the 300-IR and 500-IR doses were equally effective in reducing clinical symptoms ${ }^{(32)}$. Adverse events were related to local mucosal contact symptoms only. The 300-IR dose was recommended for clinical use, as the lower dose was associated with less adverse events. Clinical efficacy is demonstrated as early on as the first season, and studies indicate seasonal dosing protocols are effective ${ }^{(33)}$. Such efficacy and safety has also been confirmed in the paediatric population 5 to 17 years of age ${ }^{(34)}$. Thus treatment is commenced 4 months before the grass pollen season and stopped only at the end of the season for a total of 3 seasons but can be extended to 5 seasons if needed. Unlike Grazax, it does not have to be taken all year around which is advantageous in terms of patient convenience and overall cost.

\section{Droplet formulations}

Droplet formulations have been the mainstay of SLIT so far. In the meta-analysis by Wilson and colleagues, evaluating the efficacy and safety of SLIT ${ }^{(17)}$, nearly $50 \%$ of the studies were in relation to use of drops provided by the Staloral ${ }^{\circ}$ range of allergen extract products (Stallergenes, France). There are four studies in relation to grass pollen ${ }^{(35-38)}$, three in relation to tree pollen ${ }^{(39-41)}$ and three studies with weed extract ${ }^{(42-44)}$ that confirm clinical efficacy in allergic rhinitis. With HDM, two studies show clinical efficacy ${ }^{(45,46)}$, but one study clearly failed to show any difference from the placebo arm ${ }^{(47)}$. In the most recent study over 12 months and then open-label treatment for a further year in the active group, the authors demonstrated clinical improvement at 12 months $(p<0.05$ for rhinitis symptoms and $p<$ 0.001 for QOL), which was maintained at 24 months ${ }^{(46)}$. Similarly, significant improvement in QOL was seen at both time points ( $p<0.001$ and $p<0.01$, respectively). The clinical improvement was associated with increased levels of markers of immunological tolerance (IL-10 and TGF- $\beta_{1}$ ) along with decreased levels of inflammatory cytokines such as IL-13/IL-4. Overall, the studies related to droplet formulation have not been as robust as studies performed with tablet preparations, but still suggest clinical efficacy when administered to appropriately selected patients. 


\section{SLIT safety}

It is generally accepted that SLIT is safe. Evaluation of sixty studies in the recent Cochrane meta-analysis reported local sublingual/oral side-effects as the main adverse event and were only mild to moderate in severity ${ }^{(21)}$. Buccal pruritus and non-specified oral symptoms were reported as 1.6 and 2.1 total events per patient, whilst labial oedema (0.09 total events per patient), buccolingual oedema (0.22) and throat irritation (0.3) were also present. Systemic adverse events were not life threatening, ranging from skin itch (0.04), urticaria (0.03) and gastrointestinal symptoms (0.14) to rhinitis (1.45), conjunctivitis (2.95), asthma (0.1) and cough (0.93). None required intervention and were often self-limiting within a few weeks of undertaking continuous daily SLIT. Importantly, there were no reports of anaphylaxis in this series. There have, however, been case reports of SLIT induced anaphylaxis, and often it was when there was either protocol deviation ${ }^{(48)}$ or when individuals with systemic reactions to the same allergen during SCIT underwent SLIT ${ }^{(49)}$. Consensus documents recommend that SLIT should only be prescribed by individuals with training in allergy medicine. Resuscitation protocols and equipment should be in place. All patients should be educated as to when to withhold self-administration of SLIT, what to do if unplanned interruption of treatment should occur and on the management of adverse events. Detailed evaluation during therapy is important to confirm that treatment is effective to justify continuation of treatment.

\section{Conclusion}

SLIT is an alternative option to SCIT in the treatment of allergic rhinitis. The current formulations are effective, considered safe and easy to administer. Like SCIT, SLIT can immunologically alter the course of the disease long-term, leading to sustained benefit on stopping treatment. Patients need to balance daily treatment with SLIT against monthly injections with SCIT and make an informed choice on type of therapy. It must be remembered that serious adverse events have also been reported with SLIT. Thus detailed characterisation of an individual with allergic rhinitis is essential prior to commencement of immunotherapy to ensure treatment is indicated. The benefits of treatment must always outweigh any risk.

\section{Conflict of interest}

Both HHK and GR have received support to attend national and international meetings by ALK. DSR is a paid consultant for Laboratorios Leti SL, Barcelona, Spain.

\section{References}

1. Bousquet J, Khaltaev N, Cruz AA et al. Allergic Rhinitis and its Impact on Asthma (ARIA) 2008 update (in collaboration with the World Health Organization, GA(2)LEN and AllerGen). Allergy. 2008; 63 Suppl 86: 8-160.

2. Bousquet PJ, Chinn S, Janson C, Kogevinas M, Burney P, Jarvis D. Geographical variation in the prevalence of positive skin tests to environmental aeroallergens in the European Community Respiratory Health Survey I. Allergy. 2007; 62: 301-309.

3. Bousquet J, Lockey R, Malling HJ. Allergen immunotherapy: therapeutic vaccines for allergic diseases. A WHO position paper. J Allergy Clin Immunol. 1998; 102 (4 Pt 1): 558-562.

4. Malling HJ. The position of immunotherapy in the European Academy of Allergology and Clinical Immunology. J Investig Allergol Clin Immunol. 1997; 7: 356-357.

5. Canonica GW, Bousquet J, Casale T, et al Sub-lingual immunotherapy: World Allergy Organization Position Paper 2009. Allergy. 2009; 64 Suppl 91: 1-59.

6. Walker SM, Durham SR, Till SJ, et al. Immunotherapy for allergic rhinitis. Clin Exp Allergy. 2011; 41: 1177-1200.

7. Cox L, Nelson H, Lockey R, et al. Allergen immunotherapy: a practice parameter third update. J Allergy Clin Immunol. 2011; 127(1 Suppl): S1-55.

8. White $\mathrm{P}$, Smith $\mathrm{H}$, Baker N, Davis W, Frew
A. Symptom control in patients with hay fever in UK general practice: how well are we doing and is there a need for allergen immunotherapy? Clin Exp Allergy. 1998; 28 266-270.

9. Mohapatra SS, Lockey RF, Shirley S Immunobiology of grass pollen allergens. Curr Allergy Asthma Rep. 2005; 5: 381-387.

10. Frew AJ. Hundred years of allergen immunotherapy. Clin Exp Allergy. 2011; 41: 12211225

11. Lowell FC, Franklin W. A double-blind study of the effectiveness and specificity of injecton therapy in ragweed hay fever. $N$ Engl J Med. 1965; 273: 675-679.

12. Calderon MA, Alves B, Jacobson M, Hurwitz B, Sheikh A, Durham S. Allergen injection immunotherapy for seasonal allergic rhinitis. Cochrane Database Syst Rev. 2007; 1: CD001936.

13. Scadding GK, Brostoff J. Low dose sublingual therapy in patients with allergic rhinitis due to house dust mite. Clin Allergy. 1986; 16: 483-491

14. Bush RK, Swenson C, Fahlberg B, et al. House dust mite sublingual immunotherapy: results of a US trial. J Allergy Clin Immunol. 2011; 127: 974-981.

15. Nelson HS, Nolte H, Creticos P, Maloney J, Wu J, Bernstein DI. Efficacy and safety of timothy grass allergy immunotherapy tablet treatment in North American adults. Allergy Clin Immunol. 2011; 127: 72-80, 80.

16. Cox L, Compalati E, Canonica W. Will sublin- gual immunotherapy become an approved treatment method in the United States? Curr Allergy Asthma Rep. 2011; 11: 4-6.

17. Wilson DR, Torres LI, Durham SR. Sublingual immunotherapy for allergic rhinitis. Cochrane Database Syst Rev. 2003; 2: CD002893.

18. Horak F, Wheeler AW. Oral hyposensitisation with enteric-coated allergens as extension therapy following a basic subcutaneous course of injections. Int Arch Allergy Appl Immunol. 1987; 84: 74-78.

19. Bjorksten B, Moller C, Broberger U, et al. Clinical and immunological effects of oral immunotherapy with a standardized birch pollen extract. Allergy. 1986; 41: 290-295

20. Penagos M, Compalati E, Tarantini F, et al. Efficacy of sublingual immunotherapy in the treatment of allergic rhinitis in pediatric patients 3 to 18 years of age: a metaanalysis of randomized, placebo-controlled, double-blind trials. Ann Allergy Asthma Immunol. 2006; 97: 141-148.

21. Radulovic S, Calderon MA, Wilson D, Durham S. Sublingual immunotherapy for allergic rhinitis. Cochrane Database Syst Rev. 2010; 12: CD002893.

22. Calderon MA, Penagos M, Sheikh A, Canonica GW, Durham S. Sublingual immunotherapy for treating allergic conjunctivitis. Cochrane Database Syst Rev. 2011; 7 : CD007685.

23. Compalati E, Passalacqua G, Bonini M, Canonica GW. The efficacy of sublingual 
immunotherapy for house dust mites respiratory allergy: results of a GA2LEN metaanalysis. Allergy. 2009; 64: 1570-1579.

24. Shamji MH, Durham SR. Mechanisms of immunotherapy to aeroallergens. Clin Exp Allergy. 2011; 41: 1235-1246.

25. Durham SR, Yang WH, Pedersen MR Johansen N, Rak S. Sublingual immunotherapy with once-daily grass allergen tablets: a randomized controlled trial in seasonal allergic rhinoconjunctivitis. J Allergy Clin Immunol. 2006; 117: 802-809.

26. James LK, Shamji MH, Walker SM, et al. Long-term tolerance after allergen immunotherapy is accompanied by selective persistence of blocking antibodies. J Allergy Clin Immunol. 2011; 127: 509-516

27. Calderon MA, Birk AO, Andersen JS, Durham SR. Prolonged preseasonal treatment phase with Grazax sublingual immunotherapy increases clinical efficacy. Allergy. 2007; 62: 958-961.

28. Senna GE, Calderon M, Milani M. Allergy immunotherapy tablet: Grazax(R) for the treatment of grass pollen allergy. Expert Rev Clin Immunol. 2011; 7: 21-27.

29. Dahl R, Kapp A, Colombo G, et al. Sublingual grass allergen tablet immunotherapy provides sustained clinical benefit with progressive immunologic changes over 2 years. J Allergy Clin Immunol. 2008; 121: 512-518.

30. Durham SR, Emminger W, Kapp A, et al. Long-term clinical efficacy in grass polleninduced rhinoconjunctivitis after treatment with SQ-standardized grass allergy immunotherapy tablet. J Allergy Clin Immunol. 2010; 125: 131-138.

31. Durham SR. Sustained effects of grass pollen AIT. Allergy. 2011;66 Suppl 95: 50-52.

32. Didier A, Malling HJ, Worm M, et al. Optimal dose, efficacy, and safety of once-daily sublingual immunotherapy with a 5-grass pollen tablet for seasonal allergic rhinitis. J Allergy Clin Immunol. 2007; 120: 1338-1345.

33. Didier A, Worm M, Horak F, et al. Sustained 3-year efficacy of pre- and coseasonal 5-grass-pollen sublingual immunotherapy tablets in patients with grass polleninduced rhinoconjunctivitis. J Allergy Clin Immunol. 2011; 128: 559-566.

34. Wahn U, Tabar A, Kuna P, et al. Efficacy and safety of 5-grass-pollen sublingual immunotherapy tablets in pediatric allergic rhino- conjunctivitis. J Allergy Clin Immunol. 2009; 123: $160-166$

35. Sabbah A, Hassoun S, Le SJ, Andre C, Sicard $\mathrm{H}$. A double-blind, placebo-controlled trial by the sublingual route of immunotherapy with a standardized grass pollen extract. Allergy. 1994; 49: 309-313.

36. Clavel R, Bousquet J, Andre C. Clinical efficacy of sublingual-swallow immunotherapy: a double-blind, placebo-controlled trial of a standardized five-grass-pollen extract in rhinitis. Allergy. 1998; 53: 493-498

37. Smith H, White P, Annila I, Poole J, Andre C, Frew A. Randomized controlled trial of high-dose sublingual immunotherapy to treat seasonal allergic rhinitis. J Allergy Clin Immunol. 2004; 114: 831-837.

38. Ott $\mathrm{H}$, Sieber J, Brehler R, et al. Efficacy of grass pollen sublingual immunotherapy for three consecutive seasons and after cessation of treatment: the ECRIT study. Allergy. 2009; 64: 1394-1401.

39. Vourdas $D$, Syrigou E, Potamianou $P$, et al. Double-blind, placebo-controlled evaluation of sublingual immunotherapy with standardized olive pollen extract in pediatric patients with allergic rhinoconjunctivitis and mild asthma due to olive pollen sensitization. Allergy. 1998; 53: 662-672.

40. Khinchi MS, Poulsen LK, Carat F, Andre C, Hansen AB, Malling HJ. Clinical efficacy of sublingual and subcutaneous birch pollen allergen-specific immunotherapy: a randomized, placebo-controlled, double-blind, double-dummy study. Allergy, 2004; 59: 45-53.

41. Vervloet $D$, Birnbaum J, Laurent $P$, et al. Safety and efficacy of Juniperus ashei sublingual-swallow ultra-rush pollen immunotherapy in cypress rhinoconjunctivitis. A double-blind, placebo-controlled study. Int Arch Allergy Immunol. 2007; 142: 239-246.

42. La RM, Ranno C, Andre C, Carat F, Tosca MA, Canonica GW. Double-blind placebocontrolled evaluation of sublingual-swallow immunotherapy with standardized Parietaria judaica extract in children with allergic rhinoconjunctivitis. J Allergy Clin Immunol. 1999; 104(2 Pt 1): 425-432.

43. Andre C, Perrin-Fayolle M, Grosclaude M et al. A double-blind placebo-controlled evaluation of sublingual immunotherapy with a standardized ragweed extract in patients with seasonal rhinitis. Evidence for a dose-response relationship. Int Arch Allergy Immunol. 2003; 131: 111-118.

44. Bowen T, Greenbaum J, Charbonneau Y et al. Canadian trial of sublingual swallow immunotherapy for ragweed rhinoconjunctivitis. Ann Allergy Asthma Immunol. 2004; 93: 425-430.

45. Tonnel $A B$, Scherpereel $A$, Douay $B$, et al. Allergic rhinitis due to house dust mites: evaluation of the efficacy of specific sublingual immunotherapy. Allergy 2004; 59: 491-497.

46. O'Hehir RE, Gardner LM, de Leon MP, et al. House dust mite sublingual immunotherapy: the role for transforming growth factorbeta and functional regulatory T cells. Am J Respir Crit Care Med. 2009; 180: 936-947.

47. Guez S, Vatrinet C, Fadel R, Andre C. Housedust-mite sublingual-swallow immunotherapy (SLIT) in perennial rhinitis: a double-blind, placebo-controlled study. Allergy. 2000; 55: 369-375.

48. Blazowski L. Anaphylactic shock because of sublingual immunotherapy overdose during third year of maintenance dose. Allergy. 2008; 63: 374

49. de GH, Bijl A. Anaphylactic reaction after the first dose of sublingual immunotherapy with grass pollen tablet. Allergy. 2009; 64: 963-964.

Harsha H. Kariyawasam, MBBS PhD

Department of Allergy and Medical

Rhinology

Royal National Throat Nose \& Ear

Hospital

330 Gray's Inn Road

London, WC1X 8DA

England

Tel: +44-(0)207-915 1674

Fax: +44-(0)-207-915 1674

E-mail: harsha.kariyawasam@nhs.net 